\title{
Investigation of $\mathrm{SrRuO}_{3}$ barriers in SNS junctions
}

\author{
R Dömelt, C L Jia $\neq$, C Copetti†, G Ockenfuss† and A I Braginski† \\ $\dagger$ Institut für Schicht- und lonentechnik (ISI), Forschungszentrum Jülich (KFA), \\ 52425 Jülich, Germany \\ ‡ Institut für Festkörperforschung, Forschungszentrum Jülich (KFA), 52425 Jülich, \\ Germany
}

Received 7 December 1993

\begin{abstract}
High-quality films and multilayers of $\mathrm{SrRuO}_{3}$ and $\mathrm{YBa}_{2} \mathrm{Cu}_{3} \mathrm{O}_{7}(\mathrm{YBCO})$ have been grown by off-axis sputtering. High-resolution transmission electron microscopy proved that the interface is atomically sharp. sNS junctions with $\mathrm{SrRuO}_{3}$ barriers have been fabricated ex situ in an edge geometry, and in a sandwich geometry with both interfaces grown in situ. Supercurrents have been observed with barrier thicknesses from 10 to $40 \mathrm{~nm}$, and Shapiro steps could be detected. In both junction geometries the normal resistance is several orders of magnitude higher, as would be expected from the resistance of the SrRuO film, and shows a non-metallic temperature dependence. An exponential dependence of the normal resistance upon the barrier thickness has been observed.
\end{abstract}

\section{Introduction}

Metallic perovskites are a promising alternative to noble metal barrier materials in sNs junctions. Their epitaxial growth, for example on $\mathrm{YBa}_{2} \mathrm{Cu}_{3} \mathrm{O}_{7}$, makes sandwich and edge geometries possible. Also, it can be expected that the $\mathrm{S}-\mathrm{N}$ mismatch of the Fermi velocities is not as large as for silver or gold. The perovskite system $\left(\mathrm{Sr}_{1-x} \mathrm{Ca}_{x}\right) \mathrm{RuO}_{3}$ has been investigated by Char and co-workers $[1,2]$. Single crystals of the tetragonal $\mathrm{Sr}_{2} \mathrm{RuO}_{4}$ have been grown by Lichtenberg et al [3], but thin films of this phase are difficult to grow because the much more stable $\mathrm{SrRuO}_{3}$ phase is formed. sNs junctions with ferromagnetic $\mathrm{SrRuO}_{3}$ have been investigated by Antognazza et al [2]. In that work, supercurrents were observed up to a thickness of $25 \mathrm{~nm}$ and the junction resistance was determined by the SN interface, similar to SNS junctions with noble metal barriers [4]. In our work, junctions with in situ and ex situ interfaces have been investigated to distinguish between extrinsic effects due to damage in the patterning process and those due to a mismatch of the lattice constants, thermal expansion coefficients or of Fermi velocities. Furthermore, a detailed study of multilayers and the SN interface has been done by means of high-resolution transmission electron microscopy.

\section{Film growth}

Epitaxial films of $\mathrm{SrRuO}_{3}$ were grown by off-axis sputtering on $\mathrm{SrTiO}_{3}$ and $\mathrm{LaAlO}_{3}$ substrates. The x-ray patterns indicated a cubic material with a lattice constant of $3.97 \AA$. Rocking-curve widths of $0.05^{\circ}$ and Rutherford backscattering channelling minimum yields of $3 \%$ proved an excellent crystalline quality. The temperature dependence of the electric conductivity was metallic with a cusp at $150 \mathrm{~K}$ due to a decrease in the spin-flip scattering, as reported in $[2,5]$. The specific resistance of the films was $8 \times 10^{-4} \Omega \mathrm{cm}$ at $300 \mathrm{~K}$, about two orders of magnitude higher than that of $\mathrm{Au}$ or Ag. The superconducting properties of YBCO $c$-axis films grown on $\mathrm{SrRuO}_{3}$ were similar to those on the usual epitaxial substrate materials. A minimum channelling yield of $4 \%$ has been observed.

Figure 1 shows a transmission electron microscope (TEM) picture of the interface between $\mathrm{SrRuO}_{3}$ and YBCO. The interface is atomically sharp but exhibits many steps. Numerical simulations of the interface atoms show that there is no interdiffusion. The TEM results have been reported in more detail by Jia et al [6].

A serious problem for the growth of multilayers is that the high-quality $\mathrm{SrRuO}_{3}$ film allows no diffusion of oxygen at the usual oxygenating temperatures of YBCO, i.e. between 400 and $500^{\circ} \mathrm{C}$. The result is that, after the usual film annealing procedure of a trilayer, the lower YBCO film is always oxygen depleted. Figure 2 shows the $\theta-2 \theta$ scan of a trilayer of $\mathrm{YBCO}$ with a 10 $\mathrm{nm} \mathrm{SrRuO}_{3}$ interlayer. In figure 2(a), the YBCO (005) reflex consists of two peaks, which belong to a superconducting top YBCO layer with a $c$ axis of $c=11.6 \AA$ and an oxygen-deficient bottom layer with $c=11.8 \AA$. A special oxygenating procedure at temperatures between 680 and $720^{\circ} \mathrm{C}$ is needed to cause oxygen diffusion. Figure $2(b)$ shows the $(005)$ reflex of the trilayer 




Figure 1. High-resolution lattice image of the interface between $\mathrm{YBCO}$ and $\mathrm{SrRuO}_{3}$ taken along the $[100]$ direction of $Y B C O$.

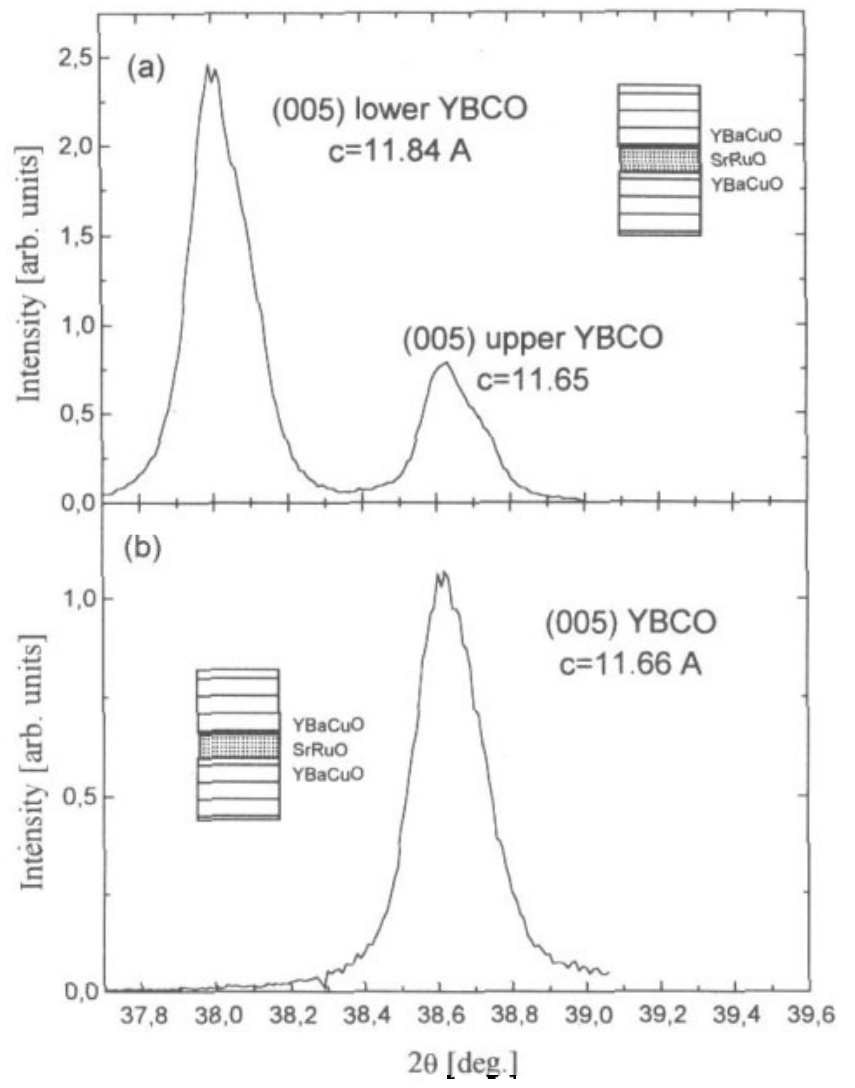

Figure 2. $\theta-2 \theta$ scan of a trilayer with a $10 \mathrm{~nm}$ thick $\mathrm{SrRuO}_{3}$ barrier; the lower layer is about $90 \mathrm{~nm}$, the upper layer about $50 \mathrm{~nm}$. (a) After the usual oxygenating procedure the lower film is oxygen depleted; (b) after $1-2 \mathrm{~h}$ at $680^{\circ} \mathrm{C}$ both films are fully oxygenated. after $1 \mathrm{~h}$ annealing in $1 \mathrm{~atm}$ oxygen at $680^{\circ} \mathrm{C}$, which indicates that both layers are properly oxygenated.

The fact that no oxygen diffuses even through an $\mathrm{SrRuO}_{3}$ film only $10 \mathrm{~nm}$ thick shows that the barrier is perfectly grown.

\section{Fabrication and measurements of SNS junctions}

Two different types of junctions have been fabricated. The first fabrication approach involved an edge junction, where the YBCO edge was etched by non-aqueous $\mathrm{Br}$-ethanol solution, using the method developed by Faley et al [7]. Figure 3 shows a scheme of the patterning process. The bottom YBCO film is etched through a mask of $\mathrm{CaO}$ and $\mathrm{ZrO}$, which is patterned by a usual resist lift-off process. The mask stays on the bottom layer during the in situ deposition of the $\mathrm{SrRuO}_{3}$ barrier and the top YBCO layer and is finally lifted-off in water (see Roas [8]) to remove the $c$-axis shunt. In the last step, bridges of 2 to $20 \mu \mathrm{m}$ width are patterned by ion beam etching (IBE).

The current-voltage $(I-V)$ characteristic of this type of junction with a nominal barrier thickness of $30 \mathrm{~nm}$ and a bridge width of $5 \mu \mathrm{m}$ is shown in figure 4 . The critical current $I_{\mathrm{c}}$ was $400 \mu \mathrm{A}$ and the normal resistance $R_{\mathrm{N}}=18 \Omega$ at $4.2 \mathrm{~K}\left(I_{\mathrm{c}} R_{\mathrm{N}}=7.2 \mathrm{mV}\right)$, determined by suppressing the supercurrent by microwave irradiation. 

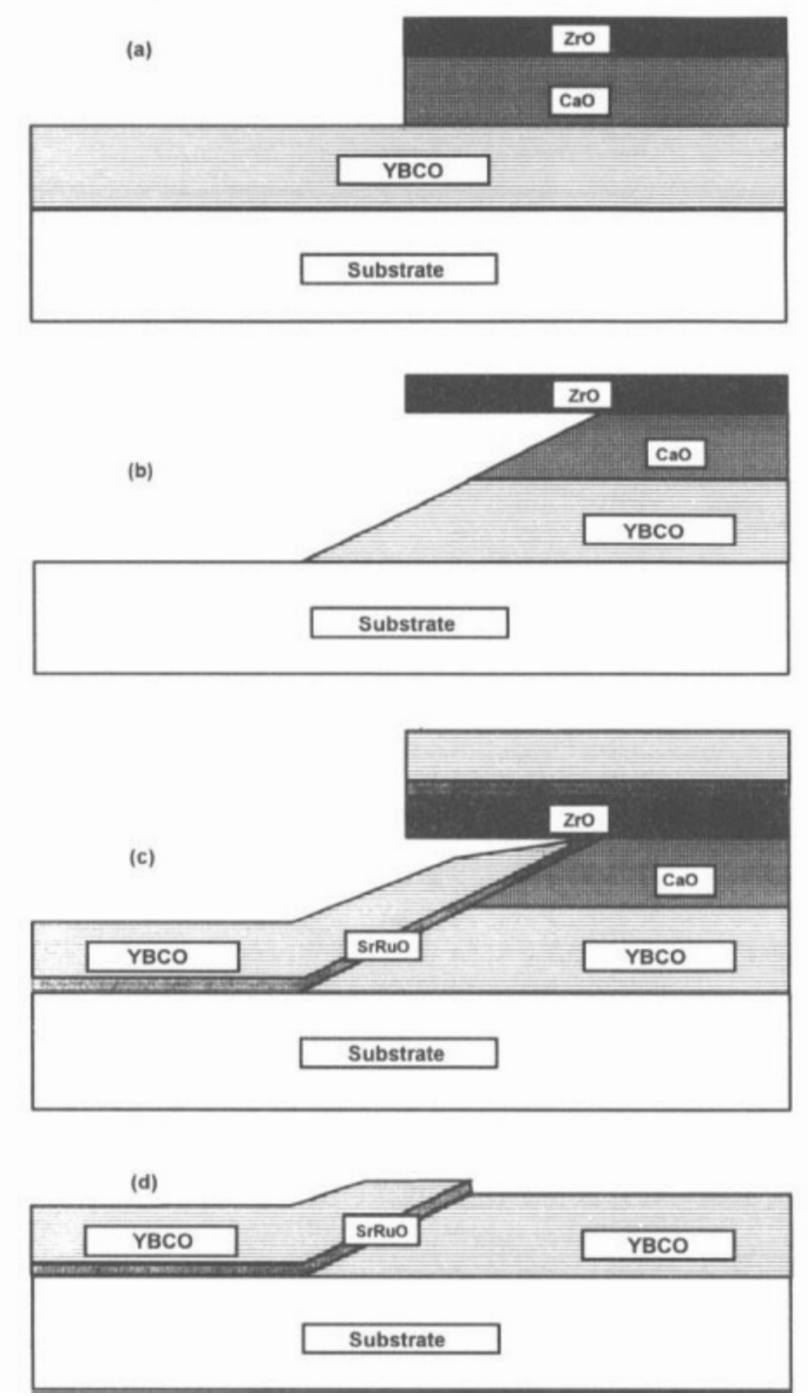

Figure 3. Fabrication of the edge-type junction: (a) a mask of $\mathrm{CaO}$ and $\mathrm{ZrO}$ is patterned by lift-otf, (b) YBCO is etched by $0.3 \% \mathrm{Br}$ in ethanol, (c) the $\mathrm{SrRuO}_{3}$ barrier and top YBCO layer are deposited, (d) $\mathrm{CaO}$ and the top layers are lifted off in $\mathrm{H}_{2} \mathrm{O}$.

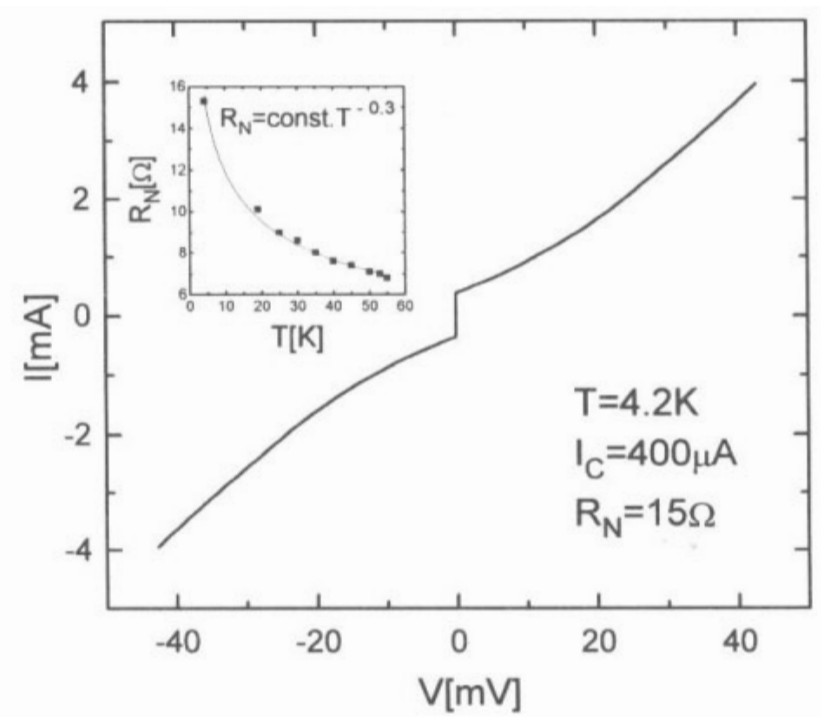

Flgure 4. $1-V$ curve of an ex situ-type junction: junction width $5 \mathrm{\mu m}$, barrier thickness $30 \mathrm{~nm}$.

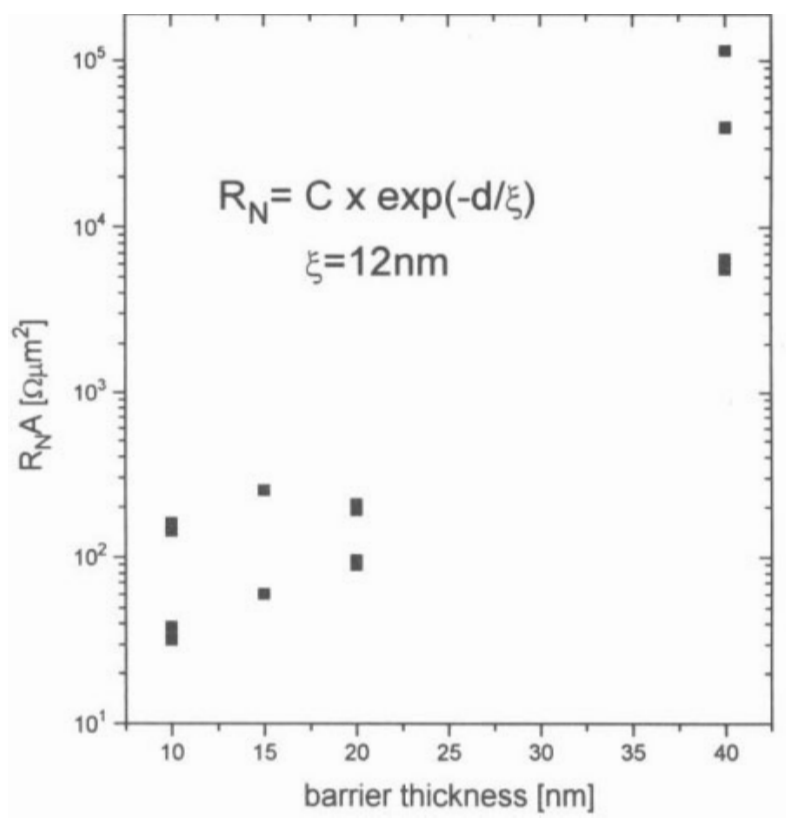

Figure 5. Thickness dependence of the normal resistance of the in situ junctions multiplied by the junction area.

Supercurrents have been observed up to $55 \mathrm{~K}$. The $R_{\mathrm{N}}$ was three orders of magnitude higher $\left(R_{\mathrm{N}} \times\right.$ junction area/barrier thickness $\simeq 0.1 \Omega \mathrm{cm}$ ) than expected from the film resistivity $\left(\rho \simeq 10^{-4} \Omega \mathrm{cm}\right)$ and, as can be seen from the inset in figure 4 , the temperature dependence was not metallic. The data for a barrier thickness of $30 \mathrm{~nm}$ can be approximated by the expression $R \propto \exp \left(T^{-0.3}\right)$, which suggests two-dimensional variable-range hopping as a possible transport mechanism for the normal current [9].

To prepare junctions with both in situ interfaces, a $c$-axis-oriented trilayer of $\mathrm{YBCO} / \mathrm{SrRuO}_{3} / \mathrm{YBCO}$ was fabricated and junction areas of $2 \mu \mathrm{m} \times(2 \mu \mathrm{m}-20$ $\mu \mathrm{m}) \times 20 \mu \mathrm{m}$ were patterned by ion beam etching. The base electrode and the sidewalls were insulated by $\mathrm{MgO}$ and the upper electrode was contacted by gold leads, which allows a three-point measurement of the SNS junctions. Junctions with barrier thicknesses from 10 to $40 \mathrm{~nm}$ were fabricated and showed supercurrents. The observation of supercurrent in this $c$-axis geometry may be due to the atomic steps at the interface (see figure 1) which allow the flow of supercurrents along the $a-b$ planes. On the other hand, supercurrents along the YBCO $c$ axis through ferromagnetic barrier materials have also been observed by Kasai et al [10]. The critical current of our junctions at $4.2 \mathrm{~K}$ varied from $5 \mu \mathrm{A}$ to $800 \mu \mathrm{A}$ at $4.2 \mathrm{~K}$, depending on the junction area and the barrier thickness. Shapiro steps have been observed above $40 \mathrm{~K}$. The normal resistance of these junctions was as high as in the edge junction geometry, and increased nearly linearly with temperature.

The critical current density for a given junction area varied by about one order of magnitude. Therefore, the $I_{c}$ data were not reliable enough to determine a dependence on the barrier thickness. However, the normal 
resistance of the junctions was much more reproducible and increased exponentially with the barrier thickness. The data could be fitted by $R_{\mathrm{N}} \sim \exp \left(-d / \xi_{\mathrm{a}}\right)$, with a characteristic length $\xi_{\mathrm{n}}$ of $12 \pm 4 \mathrm{~nm}$ (see figure 5).

\section{Discussion}

That the normal resistance of the junctions is several orders of magnitude higher than would be expected from the resistance of the $\mathrm{SrRuO}_{3}$ films has already been reported by Char and co-workers $[2,11]$ and explained by the resistance of the SN interface, which is independent of the barrier thickness. In our case the junction resistance is not determined by the interface only, since we see an exponential dependence of $R_{\mathrm{N}}$ on the barrier thickness. Furthermore, the comparison between the two types of junctions shows that the nonmetallic temperature dependence is independent of geometry and interface quality. The critical current shows no significant thickness dependence, which suggests a different transport mechanism for Cooper pairs and the normal current, but may also be explained by a random number density of atomic steps at SN interfaces. It is not yet clear if there are any pair-breaking effects due to the ferromagnetic $\mathrm{SrRuO}_{3}$ barrier. Antognazza et al [2] reported that above a thickness of $25 \mathrm{~nm}$ no critical currents could pass the barrier. In our case, the junctions with the thickness barrier $(40 \mathrm{~nm})$ showed supercurrents of about $10 \mu \mathrm{A}$ at $4.2 \mathrm{~K}$. A possible explanation could be fluctuations of the barrier thickness, which allow supercurrents only in the area where the barrier is thinner than the critical thickness. However, while TEM investigations give only a local picture, they show that the barrier thickness varies only within a few nanometres and is determined by the roughness of the lower YBCO layer and the presence of atomic steps.

\section{Acknowledgments}

We would like to thank $M$ Siegel for helpful discussions and $\mathrm{Y} \mathrm{Xu}$ and S S Ata-Allah for the preparation of the sputtering targets. This work was supported, in part, by the BMFT Consortium 'First Applications of HTS in Micro- and Cryoelectronics', project Nos 13N5811 and $13 \mathrm{~N} 5812$.

\section{References}

[1] Char K, Colclough S, Geballe T H and Myers K E 1993 Appl. Phys. Lett. 62197

[2] Antognazza I, Char K and Geballe T H 1993 Appl. Phys. Lett. 637

[3] Lichtenberg F, Catana A, Mannhart J and Schlom D G 1992 Appl. Phys. Lett. 609

[4] Ono R H, Beall J A, Cromar M W, Harwey T E, Johansson M E, Reintsema C D and Rudman D A 1991 Appl. Phys. Lett. 599

[5] Wu X D, Foltyn S R, Dye R C, Coulter X and Muenchausen R E 1993 Appl. Phys. Lett. 6219

[6] Jia C L, Dömel R and Urban K 1994 Phil. Mag. Lett. submitted

[7] Faley M I, Poppe U, Soltner H, Jia C L, Siegel M and Urban K 1993 Appl. Phys. Lett. 6315

[8] Roas B 1991 Appl. Phys. Lett. 5920

[9] Mott N F and Davis E A 1979 Electronic Properties of Doped Semiconductors (Oxford: Clarendon)

[10] Kasai M, Kanke Y, Ohno T and Kozono Y 1992 Appl. Phys. 7211

[11] Char K, Antognazza L and Geballe T H 1993 Appl. Phys. Lett. 6317 